\title{
FATORES CONTINGENCIAIS E JOGOS MULTICRITERIAL: UM ESTUDO COM MUNICÍPIOS PARANAENSES
}

\author{
Clóvis Fiirst \\ Universidade Regional de Blumenau - SC \\ Rua Antônio da Veiga, 140 - Itoupava Seca - Blumenau - SC \\ cfiirst@gmail.com \\ Nelson Hein \\ Universidade Regional de Blumenau - SC \\ Rua Antônio da Veiga, 140 - Itoupava Seca - Blumenau - SC \\ hein@furb.br \\ Adriana Kroenke Hein \\ Universidade Regional de Blumenau - SC \\ Rua Antônio da Veiga, 140 - Itoupava Seca - Blumenau - SC \\ akroenke@furb.br
}

\section{RESUMO}

O estudo tem como objetivo levantar o ranking dos municípios paranaenses observando o desempenho de fatores contingenciais, sob a ótica dos jogos multicritérios. Em relação aos procedimentos metodológicos, trata-se de um estudo descritivo, documental e quantitativo. A coleta dos dados, deu-se no ano de 2018, referente ao período de 2016. A amostra final corresponde a 344 municípios, segregados em três clusters. Para formação do ranking nos diferentes clusters procedeu-se com o modelo multicriterial, por meio do auxílio do software Excel. Os resultados demonstram que fatores contingenciais como, tecnologia, estrutura e porte são determinantes para o desempenho nas posições do ranking, e que processos internos da gestão assumidos como fator Tecnologia, e investimentos em infraestrutura e pessoal despertaram atenção na relação da teoria contingencial e jogos multicritérios, principalmente nas escolhas estratégicas que devem ser observadas. As limitações dessa pesquisa, consiste na construção de cada fator contingencial, uma vez que novas variáveis podem complementar e melhorar o entendimento dos ajustes da referida teoria, o que oportuniza outras pesquisas para explicar novos fatores contextuais internos e externos, além do que expandir essas discussões na lente da Teoria Contingencial para o setor público.

Palavra-chave: Fatores Contingenciais; Jogos Multicritérios; Setor Público; Municípios paranaenses; Desempenho.

\begin{abstract}
The study aims to raise the ranking of the municipalities of Paraná, observing the performance of contingency factors, from the perspective of multi-criteria games. Regarding the methodological procedures, it is a descriptive, documentary and quantitative study. Data collection took place in 2018, referring to the period of 2016. The final sample corresponds to 344 municipalities, segregated into three clusters. For the ranking formation in the different
\end{abstract}


clusters it was proceeded with the multicriterial model, through the aid of the Excel software. The results show that contingency factors such as technology, structure and size are determinant for performance in the ranking positions, and that internal management processes assumed as a factor Technology, and investments in infrastructure and personnel attracted attention in the relationship of contingency theory and multi-criteria games., especially in the strategic choices that must be observed. The limitations of this research is the construction of each contingency factor, since new variables can complement and improve the understanding of the adjustments of the referred theory, which allows other research to explain new internal and external contextual factors, besides expanding these discussions. in the lens of Contingency Theory for the public sector.

Keywords: Contingency factors; Multicriteria games; Public sector; Municipalities of Paraná; Performance.

\section{Como Citar:}

FIIRST, Clóvis; HEIN, Nelson; HEIN, Adriana Kroenke. Fatores Contingenciais e Jogos Multicriterial: Um estudo com Municípios Paranaenses. In: SIMPÓSIO DE PESQUISA OPERACIONAL E LOGÍSTICA DA MARINHA, 19., 2019, Rio de Janeiro, RJ. Anais [...]. Rio de Janeiro: Centro de Análises de Sistemas Navais, 2019.

\section{INTRODUÇÃO}

As organizações estão sujeitas a incerteza ambiental, traduzidas por fatores contextuais (GORLA; LAVARDA, 2012). Esses fatores podem ser internos e externos a organização, e são explicados sob a lente da teoria contingencial (CHENHALL, 2003; OTLEY, 2016). Os fatores contingenciais do ambiente interno apresentam fatores pelos quais a empresa exerce controle, tais como a estrutura, tecnologia, estratégias e tamanho, enquanto os de ambiente externo a empresa não exerce controle como exemplo PIB, IDHM, dentre outros (MOURA, CAMARGO; ZANIN, 2017).

A Teoria da Contingencia, busca correlacionar o desenvolvimento de sistemas e as restrições específicas, a fim de auxiliar o gestor na busca por alcançar objetivos, e os melhores resultados para organização, essa teoria admiti, que não há uma forma administrativa padrão aplicável a toda organização e que depende da análise das contingencias, para o delineamento do melhor ajustes nos sistemas de gestão (BEUREN; MACOHON, 2011). Já a teoria dos jogos apresenta formas de modelar uma situação de interação estratégica e de analisar tais situações após sua modelagem (FIANI, 2015). Esse estudo pretende investigar o desempenho de fatores contingenciais, sob a ótica de jogos multicritérios.

Para esse estudo, abordou-se os fatores contingenciais: ambiente, tecnologia, estrutura e tamanho. Para o fator ambiente utilizou-se o Índice de Desenvolvimento Humano Municipal (IDHM), Índice Firjan de Desenvolvimento Municipal (IFDM), Densidade Demográfica (DDEM) e Produto Interno Bruto (PIB). Para o fator tecnologia, foram utilizadas métricas de Indicador da Realização da Receita Orçamentária (IRRO) e Indicador da execução orçamentária corrente (IEOC). O fator contingencial estrutura foi assumido nesse estudo como os valores de Liquidez (LQ) despesas com pessoal (DP) e investimentos (DI). E por fim, para o fator tamanho, assumiu-se, a população (POP) e a receita arrecada (REC). Além disso, o estudo utiliza jogos multicritérios como suporte para verificação das relações das variáveis entre as posições no ranking dos municípios estudados.

Nessa perspectiva, entre fatores contingenciais e a jogos multicritério, elaborou-se a seguinte questão de pesquisa: Qual o ranking dos municípios paranaenses observando o 
desempenho de fatores contingenciais, sob a ótica dos jogos multicritérios?

O estudo contribui ao abordar sob a lente dos jogos multicritério, o desempenho de fatores ambiente, tecnologia, estrutura e tamanho, inerentes a teoria contingencial. A contribuição teórica também está centrada no fato de discutir os pressupostos da contingencia e jogos multicritérios nos entes públicos, direcionados a levantar um ranking dos municípios com base nas variáveis observadas.

Ademais, as contribuições teóricas vêm ao encontro de relacionar uma teoria organizacional (contingencial) com teorias de matriz operacional de decisão (jogos), onde além de acrescentar novos elementos que podem ser visualizados em organizações públicas, também auxilia nas escolhas das estratégias que representam maior desempenho para o interesse público.

As contribuições prático/social convergem especialmente para o interesse público em geral e principalmente para os operadores da gestão municipal, pois demonstra que escolhas estratégias devem ser observadas nos quesitos de investimentos em estrutura versos quadro de pessoal, arrecadação versos desenvolvimento econômico e principalmente os cuidados que devem estar presentes no planejamento e execução orçamentária.

Os principais achados dessa pesquisa sugerem que fatores contingenciais como Tecnologia, assumido pelos indicadores (IOEC, IRRO), que correspondem aos processos internos, voltados a execução orçamentária da gestão pública são determinantes na classificação dos municípios que se apresentam nas primeiras colocações do ranking.

\section{APORTE TEÓRICO E HIPÓTESE DA PESQUISA}

A ideia da teoria da contingência, centra-se no fato de que o desempenho das organizações são relacionados aos ajustes entre o contexto organizacional e a estrutura (CADEZ; GUILDING, 2008). Com base no que expõe, Otley (2016), as pesquisas sobre a teoria avançam em termos de apresentar uma grande quantidade de contingencias que as organizações podem enfrentar.

Contudo, nota-se que não se tem um modelo ideal para todas as estratégias e circunstancias, e assim, as contingencias devem ser entendidas num contexto dinâmico, o que leva ao uso de processos que consideram mudanças decorrentes destas variáveis (BEUREN; MACOHON, 2011; OTLEY, 2016). De acordo com Donaldson (1999) não existe uma estrutura organizacional efetivamente aplicável a todas as organizações, sendo que está varia de acordo com a sua realidade, relacionados a fatores, como a estratégias e seu tamanho.

Assim, a referida teoria busca encontrar a relação dos desenvolvimentos de sistemas, com base nos fatores internos e externos (BEUREN; MACOHON, 2011). A abordagem da teoria contingencial foi influenciada pela investigação de processos estruturais das organizações, com enfoque sistêmico, onde se consideram os fatores do ambiente no qual as organizações estão inseridas, pressupondo que as organizações são influenciadas, pelo ambiente em que operam (MOURA; CAMARGO; ZANIN, 2017).

De acordo com Chenhall (2003), pesquisas baseadas em contingencias, abordam os sistemas de controle, com a visão de que gestores, buscam adaptarem suas operações a mudanças contingenciais, com vistas a melhoria do desempenho organizacional.

Ao tratar das pesquisas que buscam utilizar a teoria contingencial nas pesquisas sobre orçamento, Gorla e Lavarda (2012), destacaram as variáveis contingenciais mais utilizadas, com destaques para estrutura, tamanho, ambiente, estratégias e tecnologia.

A teoria dos jogos, por sua vez, possibilita criar explicações para variados fenômenos sociais, admitindo diferentes modelos de acordo com as interações analisadas, assim, a essa teoria é um relevante guia em situações de interação estratégica (FIANI, 2015). Com base no 
referido autor, a teoria dos jogos, auxilia na compreensão das decisões de agentes em interação e auxilia no desenvolvimento da capacidade de raciocinar estrategicamente.

Para tanto, na teoria dos jogos observa-se que um "jogador" não depende apenas de suas estratégias, mas está condicionado, também, as estratégias dos demais, tornando o jogo um problema além da otimização, onde se leva em consideração as decisões dos outros (SMITH, 1982). Assim, o jogo pode ser compreendido como as interações estratégicas entre agentes que agem racionalmente (FIANI, 2015).

Nesse contexto da teoria dos jogos e dos fatores que interagem, o foco do estudo se concentra-se nos fatores contingenciais: ambiente; tecnologia; estrutura e porte conforme apresentado na Figura 1, a seguir:

\section{Figura 1- Desenho da pesquisa}

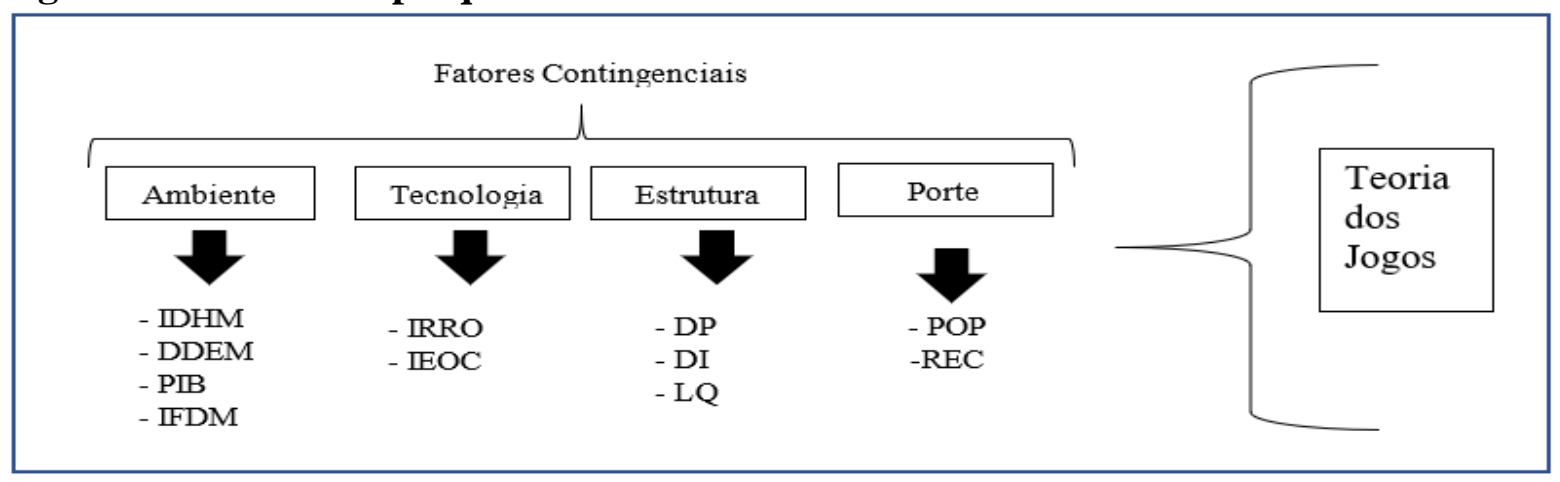

Fonte: Dados da pesquisa (2019)

Para o grupo contingencial ambiente, utilizou-se as variáveis de Índice de desenvolvimento humano municipal, variável centrada em pessoas, nas suas capacidades, oportunidades, seu bem-estar compostas pelas dimensões de saúde, educação e renda (ÍNDICE DE DESENVOLVIMENTO HUMANO MUNICIPAL BRASILEIRO, 2013). Outro fator contingencial relativo a ambiente, é o Produto Interno Bruto (PIB), assumido, baseado no pressuposto de que quanto maior o PIB maiores os recursos municipais (MARINHO, 2003). Além destas, utilizou-se a Densidade Demográfica (DDEM), que demonstra a distribuição da população pelo território (SOARES et al., 1999), e por fim, o Índice FIRJAN de desenvolvimento municipal (IFDM), que consiste numa avaliação do desenvolvimento social dos municípios, considerando fatores de emprego e renda, Saúde e educação (SILVA et al., 2013).

A tecnologia consiste nos processos de trabalho das organizações e incluem máquinas e ferramentas, materiais, pessoas, software e conhecimento (CHENHALL, 2003). Nos entes públicos o Indicador da Realização da Receita Orçamentária (IRRO), que demonstra o sucesso do planejamento e a estimativa da receita municipal e Indicador da execução orçamentária corrente (IEOC) possibilitam o acompanhamento dos processos, que demonstra a relação entre as despesas e receitas correntes (SANTOS; ALVES, 2011).

O fator Estrutura, pode ser observado nos entes públicos, a partir de métricas de investimentos e de gastos com pessoal, assim como os índices de liquidez dos entes públicos. Macedo e Corbari (2009), testaram essas variáveis, influenciando o endividamento municipal, destacando o comprometimento da receita com gastos de pessoal, as despesas de investimentos e o comprometimento patrimonial e as condições de liquidez da Administração Pública. Tais fatores, relacionam-se as condições estruturais dos entes públicos.

Por fim, as métricas de receita e a população municipal, dão a noção do tamanho dos entes públicos. As variáveis de porte associam-se a controles sofisticados, maiores recursos e maior complexidade (CADEZ; GUILDING, 2008) 
Diante das exposições feitas e com base nos fatores contingenciais assumidos na pesquisa, formulou-se a seguinte hipótese:

H1: Quanto maior for os indicadores dos fatores contingenciais, melhor é a classificação dos municípios no ranking dos jogos multicritérios.

\section{PROCEDIMENTOS METODOLÓGICOS}

\subsection{DELINEAMENTO DA PESQUISA, AMOSTRA E COLETA DE DADOS}

O delineamento metodológico assumido quanto aos objetivos é descritivo (observa o fenômeno sem manipular); documental quanto aos procedimentos (dados de fonte secundária) e quantitativo em relação a abordagem do problema (uso de técnicas estatísticas para responder o problema). A população da pesquisa é de 399 municípios, no entanto, foram excluídos 55 por não apresentarem dados suficientes a esse estudo. Portanto, a amostra final corresponde a 344 municípios paranaenses, distribuídos em três cluster pelo método $k$ média, sendo o primeiro composto por 155 municípios, o segundo com 55 municípios e o terceiro com 132 municípios. Os softwares utilizados para auxiliar tanto o tratamento, como a análise dos dados, foram o PLM 3.0, SPSS versão 21 e Microsoft Excel 2016.

Os dados foram coletados no ano civil de 2018 no portal eletrônico do Tribunal de Contas do Estado do Paraná (TCE-PR) e do Instituto Brasileiro de Geografia e Estatística (IBGE), correspondente ao exercício de 2016, visto que para as variáveis utilizadas nessa pesquisa o referido ano apresentou-se com dados auditados e previstos nas demonstrações econômicas e financeiras, bem como as informações de caráter econômico social, os anos de 2017 e 2018, ainda apresentavam-se com ausência de dados e parte pendente de auditoria.

\subsection{VARIÁVEIS E PROCEDIMENTOS DE ANÁLISES}

A operacionalização das variáveis empregadas na presente pesquisas foram descritas no Quadro 01, com o intuito de atender as dimensões contingenciais tratadas nessa pesquisa.

Quadro 1 - Variáveis da pesquisa

\begin{tabular}{|c|c|c|c|}
\hline \multicolumn{2}{|c|}{ Variáveis } & Mensuração & Literatura \\
\hline \multirow{4}{*}{ 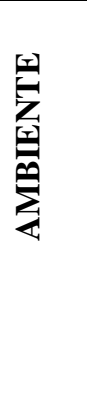 } & IFDM & $\begin{array}{l}\text { Índice FIRJAN de desenvolvimento municipal, considera emprego, } \\
\text { renda, saúde e educação }\end{array}$ & \\
\hline & IDHM & $\begin{array}{l}\text { Índice que representa o grau de desenvolvimento humano dos } \\
\text { municípios, tendo como base a longevidade, educação e renda }\end{array}$ & \\
\hline & DDEM & Densidade Demográfica - distribuição da população pelo território & \\
\hline & PIB & $\begin{array}{l}\text { Representa a soma (em valores monetários) de todos os bens e } \\
\text { serviços finais produzidos em um determinado município. Ln }\end{array}$ & \\
\hline 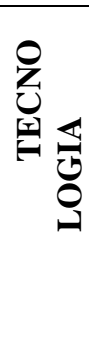 & IRRO & $\begin{array}{l}\text { Evidencia o grau de acerto do planejamento e da estimação das } \\
\text { receitas, considerando a receita total realizada e a receita total } \\
\text { orçada: } \\
\qquad I R R O=\frac{R T R}{R T O} \\
\text { Onde: } \text { IRRO = Indicador da realização da receita orçamentária; } \\
\text { RTR = Receita Total Realizada; } \mathrm{RTO}=\text { Receita total orçada. }\end{array}$ & \\
\hline
\end{tabular}




\begin{tabular}{|c|c|c|c|}
\hline & \multicolumn{3}{|c|}{$\begin{array}{l}\text { XIX SIMPÓSIO DE PESQUISA OPERACIONAL E LOGISTICA DA MARINHA } \\
\text { RIO DE JANEIRO, RJ, BRASIL - O6 A O8 DE NOVEMBRO DE } 2019\end{array}$} \\
\hline & IEOC & $\begin{array}{l}\text { Evidencia a capacidade do órgão público em manter suas despesas } \\
\text { correntes através das respectivas receitas correntes para o mesmo } \\
\text { período: } \\
\qquad I E O C=\frac{R C R}{D C R} \\
\text { Onde: IEOC = Indicador da execução orçamentária corrente; RCR } \\
=\text { Receita corrente realizada; DCR = Despesa corrente realizada. }\end{array}$ & \multirow[t]{5}{*}{$\begin{array}{l}\text { Macedo e Corbari (2009) } \\
\text { Chenhall (2003) } \\
\text { Cadez e Guilding (2008) }\end{array}$} \\
\hline \multirow{2}{*}{ 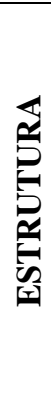 } & DP & $\begin{array}{l}\text { Indicador de Despesas com Pessoal: refere-se à parcela das receitas } \\
\text { correntes comprometidas com despesas de pessoal. Sendo: } \\
\text { DP = (Pes + Enc) / RCL } \\
\text { Onde: Pes = Pessoal; Enc = Encargos; RCL = Receita Corrente } \\
\text { Líquida. }\end{array}$ & \\
\hline & DI & $\begin{array}{l}\text { Indicador de Despesas com Investimento: apresenta a relação entre } \\
\text { as Despesas de Investimento e a Receita Corrente Líquida: Sendo: } \\
\qquad \text { DI = GI / RCL } \\
\text { Onde: GI = Gastos de Investimento; RCL = Receita Corrente } \\
\text { Líquida. }\end{array}$ & \\
\hline \multirow{2}{*}{ 됭 } & POP & Porte medido pelo Logaritmo natural da população & \\
\hline & REC & Porte medido pelo Logaritmo natural do total da receita arrecadada & \\
\hline
\end{tabular}

Fonte: Dados da pesquisa (2019).

Os indicadores são interpretados como "quanto maior, melhor" e, portanto, utilizouse cada indicador aplicando-se: $x_{i j}=\frac{x_{i j}-x_{i j}^{\min }}{x_{i j}^{\max }-x_{i j}^{\min }}$

A solução de cada jogo apresenta um vetor de estratégias $\underline{x}^{*}=\left[x_{1}, x_{2}, x_{3}, \ldots, x_{n}\right]^{t}$ representando a adoção das estratégias puras $\left(x_{i}=1\right)$ ou mistas $\left(\sum_{i=1}^{n} x_{i}=1\right)$.

Para obtenção do ranking faz-se necessários $n$ rodadas. No presente estudo optou-se em retirar os municípios que apresentam solução em cada rodada, de modo que o ranking se estabelece a partir dos maiores valores apresentados para cada município. Os demais municípios que não apresentam solução na rodada passam a compor o novo jogo e são considerados nas rodadas seguintes.

$\mathrm{Na}$ sequência, obteve-se o jogo multicriterial para cada um dos três grupos, totalizando 3 modelos. A obtenção dos rankings seguiu o exposto acima e o modelo foi organizado conforme segue:

$$
\begin{gathered}
\max v_{1}+v_{2}+v_{3}+v_{4} \\
\text { S.a } x^{t} A(s) \geq\left(v_{1}, \ldots v_{4}\right) ; s=1, \ldots, k ; k=1, \ldots, n \\
\sum_{\substack{n \\
x_{i}=1}}^{x_{i}=1} x_{i} \geq 0
\end{gathered}
$$

Estudos como de Kroenke, et al (2018) e Cecon, Kroenke, Hein (2017), também aplicaram a teoria dos jogos para decisões operacionais em organizações, o que justifica sua utilização na presente pesquisa, porém, neste caso, específico com organizações do setor público. 


\section{RESULTADOS DA PESQUISA}

\subsection{APRESENTAÇÃO E ANÁLISE DOS DADOS}

A partir dos jogos realizados, elaborou-se as Tabela 1, 2 e 3, no sentido de evidenciar as dez primeiras colocações, as dez intermediárias e as dez últimas de cada cluster formado, essa estratificação, deve-se ao fato que cada matriz de jogo gerou diversas rodadas, para tanto, essa síntese servirá de base na análise dos dados.

Tabela 1 - Modelo Geral Multicritério Cluster 1 (155)

\begin{tabular}{|c|c|c|c|c|}
\hline Município & Posição & Valor & $\mathbf{Z}$ & Estratégia \\
\hline \multicolumn{5}{|c|}{ DEZ PRIMEIROS } \\
\hline Santa Helena & $1^{\mathrm{a}}$ & $x_{125}=0,573$ & 1,68 & Mista \\
\hline Boa Vista da Aparecida & $2^{a}$ & $x_{16}=0,427$ & 1,68 & Mista \\
\hline Bandeirantes & $3^{a}$ & $x_{10}=0,748$ & 1,43 & Mista \\
\hline Mandaguaçu & $4^{\mathrm{a}}$ & $x_{65}=0,252$ & 1,43 & Mista \\
\hline Ivaiporâ & $5^{a}$ & $x_{51}=0,790$ & 1,39 & Mista \\
\hline Santa Fé & $6^{a}$ & $x_{124}=0,210$ & 1,39 & Mista \\
\hline Nova Esperança & $7^{a}$ & $x_{92}=0,889$ & 1,35 & Mista \\
\hline Quedas do Iguaçu & $8^{a}$ & $x_{109}=0,111$ & 1,35 & Mista \\
\hline Assis Chateaubriand & $9^{a}$ & $x_{7}=0,612$ & 1,33 & Mista \\
\hline Guaratuba & $10^{\mathrm{a}}$ & $x_{45}=0,388$ & 1,33 & Mista \\
\hline
\end{tabular}

(...) posição $11^{\mathrm{a}}$ a $72^{\mathrm{a}}$

\section{DEZ INTERMEDIÁRIOS}

\begin{tabular}{l|l|l|l|l}
\hline Ivatuba & $73^{\mathrm{a}}$ & $x_{52}=0,223$ & 0,92 & Mista \\
\hline Douradina & $74^{\mathrm{a}}$ & $x_{74}=0,866$ & 0,91 & Mista \\
\hline Missal & $75^{\mathrm{a}}$ & $x_{77}=0,134$ & 0,91 & Mista \\
\hline Juranda & $76^{\mathrm{a}}$ & $x_{59}=0,912$ & 0,89 & Mista \\
\hline Vitorino & $77^{\mathrm{a}}$ & $x_{155}=0,088$ & 0,89 & Mista \\
\hline Santa Isabel do Ivaí & $78^{\mathrm{a}}$ & $x_{126}=0,945$ & 0,89 & Mista \\
\hline Enéas Marques & $79^{\mathrm{a}}$ & $x_{76}=0,055$ & 0,89 & Mista \\
\hline Jaguapitã & $80^{\mathrm{a}}$ & $x_{54}=0,569$ & 0,89 & Mista \\
\hline Mercedes & $81^{\mathrm{a}}$ & $x_{74}=0,431$ & 0,89 & Mista \\
\hline Paranacity & $82^{\mathrm{a}}$ & $x_{92}=0,830$ & 0,88 & Mista
\end{tabular}

\section{(...) posição $8^{\mathrm{a}}$ a $145^{\mathrm{a}}$}

\section{DEZ ÚLTIMOS}

\begin{tabular}{l|l|l|l|l}
\hline Virmond & $146^{\mathrm{a}}$ & $x_{154}=0,313$ & 0,54 & Mista \\
\hline São Manoel do Paraná & $147^{\mathrm{a}}$ & $x_{142}=0,516$ & 0,51 & Mista \\
\hline São José das Palmeiras & $148^{\mathrm{a}}$ & $x_{141}=0,484$ & 0,51 & Mista \\
\hline Arapuã & $149^{\mathrm{a}}$ & $x_{5}=0,631$ & 0,50 & Mista \\
\hline Mirasselva & $150^{\mathrm{a}}$ & $x_{76}=0,369$ & 0,50 & Mista \\
\hline Mirador & $151^{\mathrm{a}}$ & $x_{75}=1$ & 0,43 & Pura \\
\hline Santo Inácio & $152^{\mathrm{a}}$ & $x_{198}=1$ & 0,40 & Pura \\
\hline Marumbi & $153^{\mathrm{a}}$ & $x_{72}=1$ & 0,39 & Pura \\
\hline Nossa Senhora das Graças & $154^{\mathrm{a}}$ & $x_{80}=1$ & 0,33 & Pura \\
\hline Nova Aliança do Ivaí & $155^{\mathrm{a}}$ & $x_{91}=1$ & 0,21 & Pura \\
\hline
\end{tabular}

Fonte: Dados da Pesquisa (2019)

Percebe-se, que nos três clusters gerados as variáveis contingenciais (Quadro 1) que possuíam maiores valores foram base para discriminar os agrupamentos (primeiro cluster 155 municípios, segundo cluster 55 municípios e terceiro cluster 132 municípios), nota-se, que as variáveis do grupo contingencial TECNOLOGIA e ESTRUTURA, apresenta-se como fatores determinantes na classificação dos municípios nos respectivos clusters.

Sequencialmente, observa-se que o primeiro cluster (155), gerou 76 rodadas de jogos, sendo 71 com estratégia mista, e 5 com estratégia pura. A estratificação exposta na 
Tabela 1, contribui para demonstrar o ranking dos municípios, dividido nos dez primeiros, dez intermediários e dez últimos, visto que o destaque para esse agrupamento foi o município de Santa Helena, tanto na questão da classificação do cluster, como também no jogo proposto, condicionado pelas restrições delineadas na modelagem operacional utilizada.

Tabela 2 - Modelo Geral Multicritério Cluster 2 (55)

\begin{tabular}{|c|c|c|c|c|}
\hline Município & Posição & Valor & $\mathbf{Z}$ & Estratégia \\
\hline \multicolumn{5}{|c|}{ DEZ PRIMEIROS } \\
\hline Guarapuava & $1^{\mathrm{a}}$ & $x_{19}=0,727$ & 1,61 & Mista \\
\hline São José Dos Pinhais & $2^{a}$ & $x_{45}=0,276$ & 1,61 & Mista \\
\hline Telêmaco Borba & $3^{a}$ & $x_{50}=0,775$ & 1,38 & Mista \\
\hline Terra Boa & $4^{\mathrm{a}}$ & $x_{51}=0,224$ & 1,38 & Mista \\
\hline Londrina & $5^{a}$ & $x_{27}=0,784$ & 1,31 & Mista \\
\hline Goioerê & $6^{a}$ & $x_{18}=0,156$ & 1,31 & Mista \\
\hline Maringá & $7^{a}$ & $x_{30}=0,059$ & 1,31 & Mista \\
\hline Fazenda Rio Grande & $8^{a}$ & $x_{16}=0,708$ & 1,21 & Mista \\
\hline Pinhais & $9^{a}$ & $x_{\mathrm{g} 7}=0,291$ & 1,21 & Mista \\
\hline Francisco Beltrão & $10^{\mathrm{a}}$ & $x_{17}=0,653$ & 1,13 & Mista \\
\hline
\end{tabular}

\section{(...) posição $11^{\mathrm{a}}$ a $22^{\mathrm{a}}$}

DEZ INTERMEDIÁRIOS

\begin{tabular}{l|l|c|l|l}
\hline Irati & $23^{\mathrm{a}}$ & $x_{21}=0,879$ & 0,92 & Mista \\
\hline Paranavaí & $24^{\mathrm{a}}$ & $x_{35}=0,120$ & 0,92 & Mista \\
\hline Umuarama & $25^{\mathrm{a}}$ & $x_{54}=0,516$ & 0,91 & Mista \\
\hline Arapoti & $26^{\mathrm{a}}$ & $x_{4}=0,483$ & 0,91 & Mista \\
\hline São Mateus Do Sul & $27^{\mathrm{a}}$ & $x_{46}=0,672$ & 0,90 & Mista \\
\hline Pitanga & $28^{\mathrm{a}}$ & $x_{98}=0,327$ & 0,90 & Mista \\
\hline Palotina & $29^{\mathrm{a}}$ & $x_{94}=0,583$ & 0,90 & Mista \\
\hline Astorga & $30^{\mathrm{a}}$ & $x_{5}=0,416$ & 0,90 & Mista \\
\hline Cambé & $31^{\mathrm{a}}$ & $x_{9}=0,517$ & 0,88 & Mista \\
\hline Cambará & $32^{\mathrm{a}}$ & $x_{7}=0,482$ & 0,88 & Mista \\
\hline
\end{tabular}

(...) posição $33^{\mathrm{a}}$ a $45^{\mathrm{a}}$

DEZ ÚLTIMOS

\begin{tabular}{l|l|c|l|l}
\hline Medianeira & $46^{\mathrm{a}}$ & $x_{92}=1$ & 0,69 & Pura \\
\hline Piên & $47^{\mathrm{a}}$ & $x_{36}=1$ & 0,69 & Pura \\
\hline Santo Antônio Da Platina & $48^{\mathrm{a}}$ & $x_{44}=0,935$ & 0,65 & Mista \\
\hline Jandaia Do Sul & $49^{\mathrm{a}}$ & $x_{24}=0,064$ & 0,65 & Mista \\
\hline Dois Vizinhos & $50^{\mathrm{a}}$ & $x_{15}=0,796$ & 0,63 & Mista \\
\hline Sertanópolis & $51^{\mathrm{a}}$ & $x_{49}=0,203$ & 0,63 & Mista \\
\hline Tapejara & $52^{\mathrm{a}}$ & $x_{49}=1$ & 0,50 & Pura \\
\hline Marialva & $53^{\mathrm{a}}$ & $x_{29}=1$ & 0,40 & Pura \\
\hline Loanda & $54^{\mathrm{a}}$ & $x_{26}=1$ & 0,35 & Pura \\
\hline Coronel Vivida & $55^{\mathrm{a}}$ & $x_{14}=1$ & 0,31 & Pura \\
\hline
\end{tabular}

Fonte: Dados da pesquisa (2019)

No segundo agrupamento (55 municípios), foram 29 rodadas de jogos, sendo 22 com estratégia Mista e 7 com estratégia Pura, em especial esse cluster estão os municípios com classificação média em relação aos fatores contingenciais. O município de Guarapuava (Tabela 2) apresentou com melhor classificação nesse agrupamento intermediário.

Tabela 3 - Modelo Geral Multicriterial Cluster 3 (132)

\begin{tabular}{|c|c|c|c|c|}
\hline Município & Posição & Valor & $\bar{Z}$ & Estratégia \\
\hline \multicolumn{5}{|c|}{ DEZ PRIMEIROS } \\
\hline Piraquara & $1^{\mathrm{a}}$ & $x_{g g}=0,547$ & 1,55 & Mista \\
\hline Mauá Da Serra & $2^{a}$ & $x_{95}=0,297$ & 1,55 & Mista \\
\hline Nova Cantu & $3^{a}$ & $x_{86}=0,154$ & 1,55 & Mista \\
\hline
\end{tabular}



RIO DE JANEIRO, RJ, BRASIL - 06 A 08 DE NOVEMBRO DE 2019

\begin{tabular}{|c|c|c|c|c|}
\hline Ouro Verde Do Oeste & $4^{\mathrm{a}}$ & $x_{91}=0,549$ & 1,27 & Mista \\
\hline Rio Azul & $5^{\mathrm{a}}$ & $x_{112}=0,437$ & 1,27 & Mista \\
\hline Candói & $6^{\mathrm{a}}$ & $x_{27}=0,012$ & 1,27 & Mista \\
\hline Mandirituba & $7^{\mathrm{a}}$ & $x_{77}=0,538$ & 1,20 & Mista \\
\hline Inácio Martins & $8^{a}$ & $x_{59}=0,461$ & 1,20 & Mista \\
\hline Pinhão & $9^{\mathrm{a}}$ & $x_{98}=0,697$ & 1,17 & Mista \\
\hline Marquinho & $10^{\mathrm{a}}$ & $x_{92}=0,302$ & 1,17 & Mista \\
\hline \multicolumn{5}{|c|}{ DEZ INTERMEDIÁRIOS } \\
\hline Cafeara & $62^{\mathrm{a}}$ & $x_{20}=1$ & 0,81 & Pura \\
\hline Manoel Ribas & $63^{a}$ & $x_{79}=1$ & 0,80 & Pura \\
\hline Palmital & $64^{\mathrm{a}}$ & $x_{92}=1$ & 0,80 & Pura \\
\hline Clevelândia & $65^{\mathrm{a}}$ & $x_{31}=1$ & 0,79 & Pura \\
\hline Campina Da Lagoa & $66^{\mathrm{a}}$ & $x_{22}=1$ & 0,78 & Pura \\
\hline Teixeira Soares & $67^{\mathrm{a}}$ & $x_{126}=1$ & 0,78 & Pura \\
\hline Itaguajé & $68^{\mathrm{a}}$ & $x_{64}=0,831$ & 0,78 & Mista \\
\hline Francisco Alves & $69^{a}$ & $x_{47}=0,168$ & 0,78 & Mista \\
\hline Barbosa Ferraz & $70^{\mathrm{a}}$ & $x_{12}=1$ & 0,76 & Pura \\
\hline Ângulo & $71^{\mathrm{a}}$ & $x_{g}=1$ & 0,76 & Pura \\
\hline \multicolumn{5}{|c|}{ DEZ ÚLTIMOS } \\
\hline Japira & $123^{\mathrm{a}}$ & $x_{70}=1$ & 0,45 & Pura \\
\hline Santo Antônio Do Paraíso & $124^{\mathrm{a}}$ & $x_{117}=1$ & 0,44 & Pura \\
\hline Anahy & $125^{\mathrm{a}}$ & $x_{66}=1$ & 0,44 & Pura \\
\hline Diamante D'oeste & $126^{\mathrm{a}}$ & $x_{40}=1$ & 0,42 & Pura \\
\hline Brasilândia Do Sul & $127^{\mathrm{a}}$ & $x_{19}=1$ & 0,41 & Pura \\
\hline Guaporema & $128^{\mathrm{a}}$ & $x_{54}=1$ & 0,41 & Pura \\
\hline Iracema Do Oeste & $129^{\mathrm{a}}$ & $x_{62}=1$ & 0,41 & Pura \\
\hline Ariranha Do Ivaí & $130^{\mathrm{a}}$ & $x_{12}=1$ & 0,34 & Pura \\
\hline Santa Inês & $131^{\mathrm{a}}$ & $x_{114}=0,535$ & 0,31 & Mista \\
\hline Jardim Olinda & $132^{\mathrm{a}}$ & $x_{72}=0,464$ & 0,31 & Mista \\
\hline
\end{tabular}

Fonte: Dados da Pesquisa (2019).

O último cluster (132), compõe os munícipios com valores menores em relação as variáveis contingenciais, nesse grupo foram necessárias 92 rodadas de jogos para realização do ranking, sendo 37 por estratégia mista e 55 por estratégia pura, dentre as rodadas o município de Piraquara (Tabela 3) foi o que se destacou em primeira posição nesse agrupamento, tendo como fator determinante também o grupo de variáveis pertencentes ao fatores TECNOLOGIA e ESTRUTURA.

\subsection{DISCUSSÃO DA HIPÓTESE E IMPLICAÇÕES DA PESQUISA}

A Tabela 4, demonstra um resumo das primeiras e últimas colocações em cada cluster, com o detalhamento das variáveis correspondente aos fatores (AMBIENTE TECNOLOGIA-ESTRUTURA-PORTE), assumidos da teoria contingencial.

Tabela 4 - Resumo por Cluster e Grupo Contingencial da primeira e última posição

\begin{tabular}{l|c|c|c|c|c|c|c|c|c|c|c|c|c}
\hline \multicolumn{3}{c|}{ DESCRIÇÃO } & \multicolumn{4}{c|}{ AMBIENTE } & \multicolumn{2}{c|}{ TECNOLOGIA } & \multicolumn{3}{c|}{ ESTRUTURA } & \multicolumn{3}{c}{ PORTE } \\
\hline \multicolumn{1}{c|}{ Município } & Class & cluster & IFDM & LogPiB & IDHM & DDEM & IRRO & IEOC & LQ & DP & DI & POP_Ln & rec_LN \\
\hline Santa Helena & $1^{\circ}$ & 1 & 0.73 & 4.19 & 0.74 & 30.90 & 1.51 & 1.22 & 1.61 & 0.08 & 0.30 & 10.06 & 13.61 \\
Nova Aliança Do Ivaí & $155^{\circ}$ & 1 & 0.71 & 4.54 & 0.72 & 10.90 & 1.23 & 1.02 & 2.36 & 0.12 & 0.08 & 7.27 & 13.47 \\
Guarapuava & $1^{\circ}$ & 2 & 0.79 & 4.18 & 0.73 & 53.70 & 1.53 & 1.32 & 0.98 & 0.09 & 0.14 & 12.03 & 13.66 \\
Coronel Vivida & $55^{\circ}$ & 2 & 0.75 & 4.14 & 0.72 & 31.80 & 0.78 & 1.05 & 1.41 & 0.01 & 0.01 & 9.99 & 13.58 \\
Piraquara & $1^{\circ}$ & 3 & 0.57 & 4.27 & 0.70 & 40.00 & 0.99 & 1.30 & 1.51 & 0.02 & 0.06 & 11.44 & 13.63 \\
Jardim Olinda & $132^{\circ}$ & 3 & 0.72 & 4.46 & 0.68 & 11.00 & 0.76 & 1.11 & 1.40 & 0.01 & 0.02 & 7.25 & 13.48 \\
\hline
\end{tabular}

Fonte: Dados da pesquisa (2019). 
Observa-se no fator contingencial AMBIENTE, que a variável DDEM (densidade demográfica), foi determinante para o desempenho do jogo entre os diferentes clusters, pois, sempre apresentou valores maiores nas primeiras posições, nesse mesmo sentido a variável IDHM (índice de desenvolvimento humano), também foi maior nas primeiras colocações, já as variáveis PIB (Produto Interno Bruto) e IFMD (firjan desenvolvimento municipal), não apresentaram o mesmo comportamento unânime em todos os agrupamentos.

No grupo contingencial TECNOLOGIA, as variáveis IRRO e IEOC que correspondem ao desempenho dos processos internos da execução orçamentária, foram maiores nas primeiras colocações nos três clusters, essas variáveis demonstram comportamentos de gestões eficientes, portanto, esse fator contingencial estrutura apresentase também como um determinante para o desempenho do jogo.

O fator contingencial ESTRUTURA, somente a variável DI (despesas com investimento), que representa valores despendidos em construções, compras de equipamentos, dentre outras infraestruturas, foi maior nas primeiras colocações, as demais variáveis liquidez e despesas com pessoal não obtiveram essa condição nos diferentes clusters.

As variáveis do grupo PORTE, apresentaram-se também como determinantes para o jogo, pois, tanto a POP (número da população), como a REC (volume da receita), que correspondem proxy de tamanho, foram maiores nas primeiras posições do ranking, essas duas variáveis representam principalmente a arrecadação dos municípios.

Em relação a hipóteses (H1: Quanto maior for os indicadores dos fatores contingenciais, melhor é a classificação dos municípios no ranking dos jogos multicritérios), observa-se claramente que tanto para o agrupamento dos clusters, como também para critério de decisão da programação operacional com base nas restrições (jogo), municípios que apresentaram maiores valores nas variáveis foram alocados nos melhores ranking, portanto é possivel confirmar a $\mathrm{H} 1$.

Adicionalmente a discussão anterior, os achados desse estudo que relaciona a Teoria contingencial (CHENHALL, 2003) e Teoria dos jogos (SMITH, 1982) levantam implicações relevantes para o contexto dos municípios pesquisados. Primeiramente, por demonstrar características determinantes para o bom desempenho do serviço público, como exemplo, as variáveis do fator contingencial TECNOLOGIA evidenciam a importância de manter a eficiência nos processos internos, relacionado a execução orçamentária, pois, isso reflete ter responsabilidade com os recursos arrecadados, além do que também demonstra equipe qualificada com as estimativas das receitas e no gerenciamento das alocações das despesas, essas característica denotam o que a teoria contingencial (CHENHALL, 2003), pressupõe como ajustes necessários para o funcionamento das organizações.

Outra implicação que os resultados dessa pesquisa revelam, especificamente relacionada com a teoria dos jogos, são as escolhas estratégias (FIANI, 2015) que os municípios devem atentar para obter um bom desempenho, o exemplo claro são as variáveis do grupo contingencial ESTRUTURA, pois não basta, construir obras, comprar equipamentos e fazer grandes investimentos por meio de despesa de capital, se não possuir um quadro qualificado de pessoal (DP) para transformar essas infraestruturas (DI) em serviços de qualidade para o interesse público. Ainda nesse grupo a variável (LQ) liquidez demonstra que o setor público não pode almejar obter sobras de recursos financeiros, pois não deve ter déficit, no entanto, também não deve ter altos superávit, pois comportamentos de sobras nos recursos públicos denotam anomalias na gestão pública e também ineficiência na alocação das despesas.

Por fim, uma última implicação desses resultados relaciona aos fatores AMBIENTE e PORTE, pois, municípios com maior tamanho medido pela arrecadação, não 
necessariamente possuem um IDHM e IFDM, favoráveis para representar um desempenho eficiente, isso mostra que o volume de arrecadação não explica ter uma gestão pública de qualidade, mas a administração correta dos recursos alavancados, refletem ações necessárias e estratégicas para o bem comum.

\section{CONSIDERAÇÕES FINAIS}

O objetivo da presente pesquisa, foi levantar o ranking dos municípios paranaenses observando o desempenho de fatores contingenciais, sob a ótica dos jogos Multicritérios. Para tanto, assumiu-se Ambiente, Tecnologia, Estrutura e Porte como fatores contingenciais. Em relação aos procedimentos metodológicos, trata-se de um estudo descritivo, documental e quantitativo. A coleta dos dados, deu-se no ano de 2018, referente ao período de 2016. A amostra final corresponde a 344 municípios, segregados em três clusters. Para formação do ranking nas rodadas dos jogos procedeu-se com o modelo multicriterial.

Os principais achados dessa pesquisa sugerem que fatores contingenciais como Tecnologia, representada pelos indicadores (IOEC, IRRO), que refletem os processos internos, voltados a execução orçamentária da gestão pública, são determinantes na classificação dos municípios que apresentam-se nas primeiras colocações, outro destaque também são as informações das variáveis do grupo contingencial Estrutura, assumido nesse estudo como investimentos em obras, instalações e pessoal (DP, DI), pois os resultados demonstram que investir em infraestrutura, sem a devida qualificação de pessoal, gera anomalias no desempenho dos serviços públicos. Observa-se também que as classificações tanto para os cluster, como para as primeiras posições seguiram uma lógica de quanto melhor o indicador, melhor foi a colocação dos municípios, confirmando a Hipóteses (H1) da pesquisa.

Em suma, os resultados desse estudo refletem implicações/contribuições práticosocial e teóricas. As contribuições prático/social convergem especialmente para o interesse público em geral e principalmente para os operadores da gestão municipal, pois demonstra que escolhas estratégias devem ser observadas nos quesitos de investimentos em estrutura versos quadro de pessoal, arrecadação versos desenvolvimento econômico e principalmente os cuidados que deve estar presente no planejamento e execução orçamentária. As contribuições teóricas vêm ao encontro de relacionar uma teoria organizacional (contingencial) com teorias de matriz operacional de decisão (jogos), onde além de acrescentar novos elementos que podem ser visualizados em organizações públicas, também auxilia nas escolhas das estratégias que representam maior desempenho para o interesse público.

As limitações dessa pesquisa, consiste na construção de cada fator contingencial, uma vez que novas variáveis podem complementar e melhorar o entendimento dos ajustes da referida teoria, o que oportuniza outras pesquisas para explicar novos fatores contextuais internos e externos, além do que expandir essas discussões na lente da Teoria Contingencial para o setor público. Uma outra limitação também é no modelo multicriterial utilizado, outros modelos podem ser utilizados para as decisões operacionais. Em relação a sugestão de estudos futuros uma oportunidade seria avaliar a teoria dos jogos com outras abordagens organizacionais como exemplo as capacidades dinâmicas, capacidades baseadas em recursos, novos achados poderiam explicar elementos que geram o desempenho para as melhores posições no ranking. 


\section{REFERÊNCIAS BIBLIOGRÁFICAS}

[1] BEUREN, Ilse; MACOHON, Edson. Institucionalização de hábitos e rotinas na contabilidade gerencial à luz da teoria da contingência: Um estudo em indústrias de móveis em São Bento do Sul. Revista de Gestão dos Países de Língua Portuguesa, v. 10, n. 1-2, p. 78-91, 2011.

[2] CADEZ, Simon; GUILDING, Chris. An exploratory investigation of an integrated contingency model of strategic management accounting. Accounting, organizations and society, v. 33, n. 7-8, p. 836-863, 2008.

[3] CECON, Bianca et al. Associação da divulgação social e ambiental com o valor de mercado: um estudo com juegos vetoriais. Anales de ASEPUMA, n. 25, p. 23, 2017.

[4] CHENHALL, Robert H. Management control systems design within its organizational context: findings from contingency-based research and directions for the future. Accounting, Organizations and Society, v. 28, n. 2, p. 127-168, 2003.

[5] DONALDSON, Lex. "Teoria da Contingência Estrutural” in Clegg, Hardy e Nord, Handbook de Estudos Organizacionais. São Paulo, Atlas, 1999, v. 1, capítulo 3.

[6] FIANI, R. Teoria dos jogos. 4.ed. Rio de Janeiro: Elsevier, 2015.

[7] GORLA, Marcello Christiano; LAVARDA, Carlos Eduardo Facin. Teoria da contingência e pesquisa contábil. Revista de Administração, Contabilidade e Economia da Fundace, v. 3, n. 2, 2012.

[8] INDICE DE DESENVOLVIMENTO HUMANO MUNICIPAL BRASILEIRO. Série Atlas do desenvolvimento Humano do Brasil, 2013.

[9] KROENKE, Adriana et al. Scalar And Vector Games In The Evaluation Of Social And Environmental Disclosure And Their Relationship With Market Value. Pesquisa Operacional, v. 38, n. 2, p. 215-246, 2018.

[10] MACEDO, Joel J.; CORBARI, Ely Célia. Efeitos da lei de responsabilidade fiscal no endividamento dos municípios brasileiros: uma análise de dados em painéis. Revista Contabilidade \& Finanças, v. 20, n. 51, p. 44-60, 2009.

[11] MARINHO, Alexandre. Avaliação da eficiência técnica nos serviços de saúde nos municípios do Estado do Rio de Janeiro. Revista brasileira de economia, v. 57, n. 3, p. 515-534, 2003.

[12] MOURA, Geovanne Dias de ; CAMARGO, Tiago Francisco de ; ZANIN, Antonio. Competitividade de Mercado e Gerenciamento de Resultados: um estudo sob a ótica da teoria da contingência. Revista de Ciências da Administração, v. 19, n. 49, p. 86-101, 2017.

[13] OTLEY, David. The contingency theory of management accounting and control: 19802014. Management accounting research, v. 31, p. 45-62, 2016. 
[14] SANTOS, Sandra Regina Toledo; ALVES, Tiago Wickstrom. O impacto da Lei de Responsabilidade Fiscal no desempenho financeiro e na execução orçamentária dos municípios no Rio Grande do Sul de 1997 a 2004. Revista de Administração Pública, v. 45, n. 1, p. 181-208, 2011.

[15] SILVA, Lara Lúcia da et al. A influência do desempenho tributário e gestão fiscal no Índice Firjan de Desenvolvimento (IFDM) dos municípios de Minas Gerais. Revista de Ciências Humanas, v. 13, n. 1, p. 199-219, 2013.

[16] SMITH, J. M. Evolution and the Theory of Games. Cambridge university press, 1982.

[17] SOARES, Ana Cristina Lima Gouveia et al. Índice de desenvolvimento municipal: hierarquização dos municípios do Ceará no ano de 1997. Revista Paranaense de Desenvolvimento-RPD, n. 97, p. 71-89, 2011. 